\title{
Tumor lymphangiogenesis predicts melanoma metastasis to sentinel lymph nodes
}

\author{
Soheil S Dadras ${ }^{1,2}$, Bernhard Lange-Asschenfeldt ${ }^{3}$, Paula Velasco ${ }^{3}$, Lynh Nguyen $^{1}$, \\ Anish Vora ${ }^{1}$, Alona Muzikansky ${ }^{4}$, Katharina Jahnke ${ }^{3}$, Axel Hauschild ${ }^{3}$, Satoshi Hirakawa ${ }^{1}$, \\ Martin C Mihm² and Michael Detmar ${ }^{1}$ \\ ${ }^{1}$ Cutaneous Biology Research Center and Department of Dermatology, Massachusetts General Hospital and \\ Harvard Medical School, Charlestown, MA, USA; ${ }^{2}$ Department of Pathology, Massachusetts General Hospital \\ and Harvard Medical School, Boston, MA, USA; ${ }^{3}$ Department of Dermatology, University of Kiel, Kiel, \\ Germany and ${ }^{4}$ Department of Biostatistics, Massachusetts General Hospital and Harvard Medical School, \\ Boston, MA, USA
}

\begin{abstract}
Cutaneous melanoma is a common melanocytic neoplasm that can quickly metastasize to regional lymph nodes. Currently, prognosis is determined by measuring tumor thickness but more reliable markers for metastatic spread are urgently needed. We investigated whether the extent of tumor lymphangiogenesis can predict melanoma metastasis to sentinel lymph nodes. We quantified the extent of tumor lymphangiogenesis, as well as other factors, in excised primary tumors and in sentinel lymph node biopsy samples from 45 patients with primary cutaneous melanoma. The results were correlated with histological and clinical outcome. Primary melanomas from patients whose tumors had metastasized to the sentinel lymph nodes contained prominent 'hot spots' of increased lymphatic vessel density, compared to nonmetastatic tumors. Multivariate risk analysis revealed that the lymphatic vascular area of primary melanomas, an index of tumor lymphangiogenesis, was the most sensitive prognostic marker for sentinel lymph node metastasis, and was even able to more accurately predict which tumors were metastatic to sentinel lymph nodes than the currently used method of measuring tumor thickness. Highly lymphangiogenic melanomas maintained their lymphangiogenic activity after metastasis to the sentinel lymph node. The extent of tumor lymphangiogenesis is a highly sensitive (83\%) and specific $(89 \%)$ prognostic marker of lymph node metastasis. Assessment of lymphangiogenesis in primary melanomas may be a more effective approach than the currently used technique of measuring tumor thickness in selecting patients with early metastatic disease for aggressive therapy.

Modern Pathology (2005) 18, 1232-1242. doi:10.1038/modpathol.3800410; published online 1 April 2005
\end{abstract}

Keywords: LYVE-1; lymphangiogenesis; angiogenesis; sentinel lymph node; VEGF-C and VEGF-D

In 2003 , it was estimated that 7700 patients in the US would die from cutaneous melanoma, ${ }^{1}$ a melanocytic tumor with increasing worldwide incidence and mortality rates. ${ }^{2}$ Melanoma is among the most common types of cancer in young adults, and death from melanoma occurs at a younger age than for any other common malignancy, ${ }^{3}$ representing a substantial public health problem. The prognosis of patients with melanoma depends on the tumor stage at diagnosis, and is currently based on microstaging and clinical/radiologic evaluation for metastases. In addition to a complete surgical excision with

Correspondence: Dr SS Dadras, MD, PhD, Cutaneous Biology Research Center, Massachusetts General Hospital, Building 149, 13th Street, Charlestown, MA 02129, USA.

E-mail: sdadras@partners.org

Received 22 November 2004; revised 6 February 2005; accepted 7 February 2005; published online 1 April 2005 wide margins, patients with primary melanomas $>1.0 \mathrm{~mm}$ thick undergo intraoperative lymphatic mapping and sentinel lymph node (SLN) biopsy to identify the first deposition of microscopic metastatic cells. ${ }^{4}$ Among several prognostic parameters, tumor thickness is currently the most sensitive parameter for predicting the metastatic risk of cutaneous melanoma. However, the prognosis for individual melanoma patients is still difficult to determine, since thin melanomas can also develop into lethal metastases. ${ }^{5}$ More accurate prognostic indicators for melanoma metastasis are therefore urgently needed.

Recently, studies in mouse tumor models have shown that some types of malignant tumors actively induce the formation of lymphatic vessels (lymphangiogenesis), which promotes tumor spread to regional lymph nodes. ${ }^{6,7}$ These findings strongly suggest that tumors can actively induce lymphan- 
giogenesis via production of lymphangiogenic factors such as vascular endothelial growth factor-C (VEGF-C) and VEGF-D, and that the extent of tumor lymphangiogenesis can be reliably determined by immunostains for the lymphatic endothelial hyaluronan receptor-1 (LYVE-1), a specific marker for lymphatic endothelium. ${ }^{8}$ These findings also indicate that the degree of tumor-associated lymphangiogenesis might serve as a novel predictor for lymph node metastasis. However, the relevance of these animal studies to human cancers has remained unclear. Our recent retrospective analysis of clinically and histologically matched cohorts of patients with cutaneous melanoma provided the first indication that intra- and peritumoral lymphangiogenesis was increased in melanomas that later metastasized to lymph nodes, as compared with nonmetastatic melanomas. ${ }^{9}$ Analysis of tumor lymphangiogenesis might therefore be a useful prognostic marker.

We performed a prospective investigation to determine whether the level of tumor lymphangiogenesis might predict the risk of melanoma metastasis to SLN-the earliest form of microscopic metastatic disease-using tissue samples obtained from 45 consecutive patients with cutaneous melanoma who were treated by surgical excision along with SLN biopsy.

\section{Materials and methods}

\section{Patient Population and Histological Analyses}

Between 2000 and 2003, 110 patients with cutaneous melanoma were identified at the Department of Dermatology at the University of Kiel, Germany and subjected to SLN biopsy, following the excision of primary tumors that exceeded $1 \mathrm{~mm}$ in thickness, using lymphatic scintigraphy as described. ${ }^{4} \mathrm{~A}$ total of 45 consecutive patients underwent SLN biopsies, 27 were found to have SLNs that were free of melanoma metastases (mean age of 53.8 years; range 19-76 years; termed 'SLN-negative'), whereas the tumors were found to have metastasized to the SLN in the remaining 18 patients (mean age of 54 years; range 29-78 years; termed 'SLN-positive'). These two groups were otherwise similar, in terms of tumor histologic type, presence and degree of ulceration, anatomic site, signs of regression and peritumoral inflammation (Table 1).

SLN-positive patients subsequently received adjuvant systemic therapy with recombinant interferon alpha-2a (Roferon ${ }^{\mathbb{R}}$, Hoffmann La Roche, Switzerland) or interferon alpha-2b (Intron ${ }^{\circledR}$ A, Essex, Switzerland) for up to 60 months. Compared to six out of 18 SLN-positive, only two out of 27 SLN-negative, patients developed metastases to distant lymph nodes or organs within 2 years. The diagnosis of melanoma, the tumor thickness, the level of tumor invasion, and SLN pathologic status were reconfirmed by two pathologists (SSD and MCM). Additional parameters such as the frequency
Table 1 Clinical and pathological characteristics of patients with melanoma

\begin{tabular}{|c|c|c|c|}
\hline Category & $\begin{array}{c}S L N- \\
\text { negative }\end{array}$ & $\begin{array}{c}S L N- \\
\text { positive }\end{array}$ & $\mathrm{P}$-value \\
\hline Number of patients & 27 & 18 & \\
\hline Male & 10 & 10 & \\
\hline Female & 17 & 8 & 0.24120 \\
\hline \multicolumn{4}{|l|}{ Age at diagnosis (years) } \\
\hline Mean & 54 & 53.8 & \\
\hline Range & $29-78$ & $19-76$ & 0.97243 \\
\hline \multicolumn{4}{|l|}{ Histologic type } \\
\hline Superficial spreading & 22 & 8 & \\
\hline Nodular & 4 & 8 & \\
\hline Acral lentiginous & 1 & 2 & 0.20090 \\
\hline \multicolumn{4}{|l|}{ Tumor thickness (mm) } \\
\hline$<1.5$ & 19 & 3 & \\
\hline$\geq 1.5$ & 8 & 15 & \\
\hline Mean & 1.7 & 3.01 & 0.02850 \\
\hline \multicolumn{4}{|l|}{ Level of invasion } \\
\hline I-III & 16 & 3 & \\
\hline IV-V & 11 & 15 & 0.00010 \\
\hline \multicolumn{4}{|l|}{ Ulceration } \\
\hline Present & 4 & 6 & \\
\hline Absent & 23 & 12 & \\
\hline Mean size (mm) & 4.98 & 4.42 & 0.16563 \\
\hline \multicolumn{4}{|l|}{ Anatomic site } \\
\hline Upper extremity & 8 & 2 & \\
\hline Lower extremity & 13 & 11 & \\
\hline Head and neck & 0 & 0 & \\
\hline Trunk & 6 & 5 & 0.34244 \\
\hline \multicolumn{4}{|l|}{ Mitoses $\left(\mathrm{mm}^{2}\right)$} \\
\hline$>6.0$ & 7 & 10 & \\
\hline $1-5$ & 9 & 5 & \\
\hline 0 & 9 & 3 & \\
\hline NA & 2 & 0 & 1.00000 \\
\hline \multicolumn{4}{|l|}{ Regression } \\
\hline Present & 2 & 2 & \\
\hline Absent & 23 & 15 & \\
\hline NA & 2 & 1 & 0.71531 \\
\hline \multicolumn{4}{|c|}{ Tumor infiltrating lymphocytes } \\
\hline Absent & 8 & 5 & \\
\hline Present & 13 & 10 & \\
\hline Brisk & 4 & 2 & \\
\hline NA & 2 & 1 & 0.88914 \\
\hline
\end{tabular}

$\mathrm{NA}=$ not applicable, tumor not in vertical growth phase.

of mitoses, tumor regression and ulceration were also evaluated. Peritumoral inflammation was evaluated as absent, present (non-brisk), or brisk. ${ }^{10}$

\section{Immunostains}

To analyze tumor angiogenesis and lymphangiogenesis, we performed double immunofluorescence stains of the 45 primary melanomas for the vascular marker CD31 and for the lymphatic-specific marker LYVE-1. Paraffin sections of $4 \mu \mathrm{m}$ thickness were double stained using a rabbit polyclonal antibody 
Table 2 Antibodies used for immunofluorescent analysis

\begin{tabular}{|c|c|c|c|c|}
\hline Antibody & Staining for & Source & Tуре & Dilution and conditions $s^{\mathrm{a}}$ \\
\hline LYVE-1 & Lymphatics & DG Jackson & Polyclonal & 1:600; O/N $\left(4^{\circ} \mathrm{C}\right)$ \\
\hline CD31 & Panvascular & DAKO & Monoclonal, IgG1 & $1: 50 ; 3 \mathrm{~h}(\mathrm{RT})$ \\
\hline D2-40 & Lymphatics & Signet & Monoclonal, IgG1 & 1:100; O/N $\left(4^{\circ} \mathrm{C}\right)$ \\
\hline VEGF-C & Melanoma cells, macrophages & Zymed & Polyclonal & $1: 100 ; \mathrm{O} / \mathrm{N}\left(4^{\circ} \mathrm{C}\right)$ \\
\hline VEGF-D & Melanoma cells, macrophages & M Achen & Monoclonal & 1:100; O/N ( $\left.4^{\circ} \mathrm{C}\right)$ \\
\hline
\end{tabular}

${ }^{\mathrm{a}} \mathrm{RT}=$ room temperature; $\mathrm{O} / \mathrm{N}=$ over night.

against human LYVE- $1^{8}$ and a mouse monoclonal antibody against human CD31 (1:40; DAKO), as previously described ${ }^{9,11}$ (Table 2). For additional lymphatic-specific stains, the antibody D2-40 (Signet, Dedham, MA) was used. We have recently shown that this antibody reliably and specifically recognizes human podoplanin, a mucin-type glycoprotein that is specifically expressed by lymphatic endothelium, but not by blood vascular endothelium. ${ }^{12,13}$ Cell nuclei were counterstained with Hoechst bisbenzimide (Sigma) at $20 \mu \mathrm{g} / \mathrm{ml}$. Additional immunohistochemical analysis was performed using affinity-purified rabbit polyclonal antibodies against human vascular endothelial growth factor-C (VEGF-C) and VEGF-D (kindly provided by Dr M Achen). ${ }^{14}$ For specificity controls, the primary antibodies were omitted. The immunostained sections were scored semi-quantitatively for VEGF-C and VEGF-D expression by tumor cells, in comparison to tumor infiltrating macrophages (internal positive control, $3+$ ) by researchers who were blinded to the SLN status. The results were scored as follows: $1+$ for faintly positive, $2+$ for moderately positive, $3+$ for strongly positive.

\section{Computer-Assisted Morphometric Analysis}

Paraffin sections were immunostained for CD31 and LYVE-1 as described above, and were examined using a Nikon E-600 microscope. Digital images were captured using a SPOT digital camera. For each section, three fields in the peritumoral area with the highest lymphatic vascular density ('hot spots') were evaluated at $\times 100$ magnification. Digital images of tumor-associated lymphatic vessels and blood vessels were captured in the same field. Peritumoral lymphatic vessels were defined as LYVE-1-positive vessels within an area of $100 \mu \mathrm{m}$ from the tumor border. Intratumoral lymphatic vessels were defined as LYVE-1-positive vessels located within the tumor mass and not confined by invagination of normal tissue. Tumor borders were determined on serial sections, using Hoechst nuclear stains and hematoxylin and eosin stains. Morphometric analyses of lymphatic vessels and of blood vessels were performed in the peritumoral area using the IP-Lab software (Scanalytics, Fairfax, VA, USA) to determine the vessel number per $\mathrm{mm}^{2}$, that is, lymphatic vessel density (LVD), and the blood vessel density (BVD). The average vessel size (in $\mu \mathrm{m}^{2}$ ) and the relative tissue area occupied by vessels, that is, the lymphatic vessel area (LVA; expressed as \%) and the blood vessel area (BVA), were quantified as previously described. ${ }^{9,11}$ Tumorassociated lymphatics and blood vessels were analyzed within and surrounding the primary melanomas and also in metastatic SLNs using double immunofluorescence with antibodies against CD31 and LYVE-1.

\section{Statistical Analyses}

The unpaired Student's $t$-test was used to determine the statistical significance ( $P$ value) of the mean for all vascular parameters. In a univariate analysis, we tested the association between different risk factors for melanoma metastasis to SLN, using the Fisher's exact test for dichotomous variables, the $\chi^{2}$-test for categorical variables and the Wilcoxon Rank-Sum test for continuous or ordinal variables. A multivariate logistic regression model was applied to the risk factors that were found to be significant in the univariate analysis, and then a stepwise selection method was used to select the significant predictors for SLN metastasis. Sensitivity was calculated based on the number of true positives (patients who were SLN-positive and had an LVA $>1.5 \%$ ), divided by the number of true positives plus the number of false negatives (patients who were SLN-positive but had an LVA $<1.5 \%$ ). Specificity was calculated based on the number of true negatives (patients who were SLN-negative and had an LVA $<1.5 \%$ ) divided by the number of true negatives plus the number of false positives (patients who were SLN-negative but had an LVA $>1.5 \%$ ).

\section{Results}

\section{Increased Tumor Lymphangiogenesis in Primary Melanomas of SLN-Positive Patients}

We analyzed 45 consecutive patients with nonmetastatic $(n=27)$ or with metastatic $(n=18)$ primary cutaneous melanoma to the SLN. SLN-positive

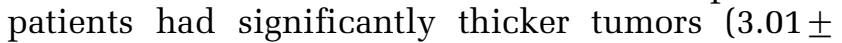
$0.52 \mathrm{~mm}$ ) than SLN-negative patients (1.70士 $0.31 \mathrm{~mm}, P=0.0285$ ) (Table 1). Histologic analysis revealed that additional prognostic parameters such 
as tumor ulceration, mitotic activity, regression and number of tumor infiltrating lymphocytes did not differ between groups (Table 1). Similarly, there was no significant difference in clinical parameters such as sex, age or anatomic site between SLN-positive and SLN-negative patients. Next, melanoma-associated lymphatic and blood vessels were simultaneously visualized in all primary melanomas, using double immunofluorescence analysis with antibodies against the lymphatic vessel marker LYVE-1 and the panvascular marker CD31. Melanomas from SLN-positive patients exhibited prominent 'hot spots' of increased lymphatic vessel density, within tumors and surrounding the tumor borders (Figure $1 \mathrm{~b}$ and $\mathrm{d}$ ), whereas only a few lymphatic vessels surrounding the tumor border were detected in SLNnegative patients (Figure 1a and c). In contrast, blood vessels were homogeneously distributed throughout the tumors in both groups.

Intratumoral lymphatics were found more frequently in primary melanomas taken from SLNpositive patients $(83.3 \pm 0.09 \%)$ than in those taken from SLN-negative patients $(59.3 \pm 0.09 \%, P=0.0960)$. Pigmented tumor cells within LYVE-1 + lymphatic vessels-that is, from tumors that have undergone lymphatic vascular invasion-were found in two out of 18 (11\%) patients with SLN-positive melanomas, but not in any patients with SLN-negative melanomas. There was a positive correlation between LVD and lymphatic vascular invasion among SLN-positive patients (LVD 26.6 \pm 6.3 lymphatic vessels $/ \mathrm{mm}^{2}$ with lymphatic vascular invasion, $15.1 \pm 1.6$ vessels $/ \mathrm{mm}^{2}$ without lymphatic vascular invasion; $P=0.0348$ ). Immunohistochemical analysis with the lymphatic-specific antibody D2-40 ${ }^{13}$ showed a strikingly similar staining pattern as experiments performed with the antibody against LYVE-1. Both of these antibodies labeled tumorassociated lymphatic vessels, but not of blood vessels (Figure 1f).

Computer-assisted morphometric analysis revealed a 2.4-fold increase in the peritumoral LVD $\left(16.4 \pm 1.7\right.$ vessels $\left./ \mathrm{mm}^{2}\right)$ and of the LVA $(2.2 \pm 0.2 \%)$ in SLN-positive melanomas, compared with SLNnegative melanomas (LVD of $7.0 \pm 1.1$ vessels $/ \mathrm{mm}^{2}$, $P=0.00022$, and LVA of $0.9 \pm 0.1 \%, P=0.00008$; Figure 2d-f). SLN-positive melanomas also showed a slight increase of BVD $\left(48.2 \pm 4.6\right.$ vessels $\left./ \mathrm{mm}^{2}\right)$ and of the BVA $(4.3 \pm 0.4 \%)$, compared with SLNnegative melanomas (BVD of $36.3 \pm 4.1$ vessels $/ \mathrm{mm}^{2}$, $P=0.03392$, and BVA of $2.9 \pm 0.2 \%, P=0.01106$; Figure 2a-c).

\section{Extent of Lymphangiogenesis can be Used to Predict Melanoma Metastasis to SLN}

To investigate whether tumor lymphangiogenesis might serve as a new prognostic indicator for SLN metastasis, we next performed a comprehensive statistical data evaluation that included univariate and multivariate analyses. A univariate analysis of the risk factors for melanoma metastasis identified peritumoral LVA as the most significant prognostic parameter, followed by peritumoral LVD, level of tumor invasion, tumor thickness, BVD and finally BVA (Table 3; Figure 2a and c). The expression level of VEGF-C, but not VEGF-D, was also a significant predictor of metastasis to the SLN (Table 3). Other clinical and histological parameters, such as age, sex, anatomic site, histologic type, regression, ulceration, lymphatic vascular invasion, presence of intratumoral lymphatics, microsatellites and tumor infiltrating lymphocytes were not found by the univariate analysis to predict the ability of melanoma to metastasize to the SLN (Table 3). Linear regression and Pearson correlation coefficient analyses showed a positive correlation between BVA and tumor thickness (borderline statistical significance; $P=0.0539$ ) but not between LVA and tumor thickness $(P=0.1515)$. This indicates that the extent of lymphangiogenesis, unlike angiogenesis, is independent of tumor thickness.

A multivariate logistic regression analysis with applied selection criteria revealed that the level of peritumoral LVA was the most significant, independent predictor of melanoma metastasis to SLN $(P=0.00037$, odds ratio $=5.527,95 \%$ confidence limits 1.740-17.552) - it was even more accurate than the currently used prognostic factor, tumor thickness. We next used a stepwise multivariate logistic regression analysis to determine whether some combination of variables would provide a better estimate of the relative risk of metastasis than any single variable. LVA by itself, however, provided the best estimate, whereas LVA and VEGF-C expression levels combined were only of borderline statistical significance $(P=0.0562$, odds ratio $=$ 4.369, 95\% confidence limits 1.478-12.912).

We next classified all patients into three groups, based on peritumoral LVA: low $(\leq 1.0 \%)$, medium $(1.0 \% \leq 1.5 \%)$ or high $(>1.5 \%)$. The great majority of patients (89\%) with low to medium LVA were free of SLN metastases, whereas $89 \%$ of those with high LVA were SLN-positive. Based on this threshold (LVA of $1.5 \%$ ), the sensitivity and specificity of peritumoral LVA in predicting metastasis to the SLN were calculated to be 83 and $89 \%$, respectively. Importantly, six out of 18 SLN-positive patients developed distant lymph node and/or organ metastases, and $83 \%$ of these patients had an LVA $>1.5 \%$. So the extent of peritumoral lymphangiogenesis appears to be a highly significant, novel predictor of melanoma spread to SLN. This is the earliest form of melanoma metastasis, and can currently only be detected by microscopic analysis of lymph node biopsy samples.

\section{Increased VEGF-C Expression in SLN-Positive Melanomas}

As VEGF-C and VEGF-D have been shown to regulate tumor lymphangiogenesis in experimental 

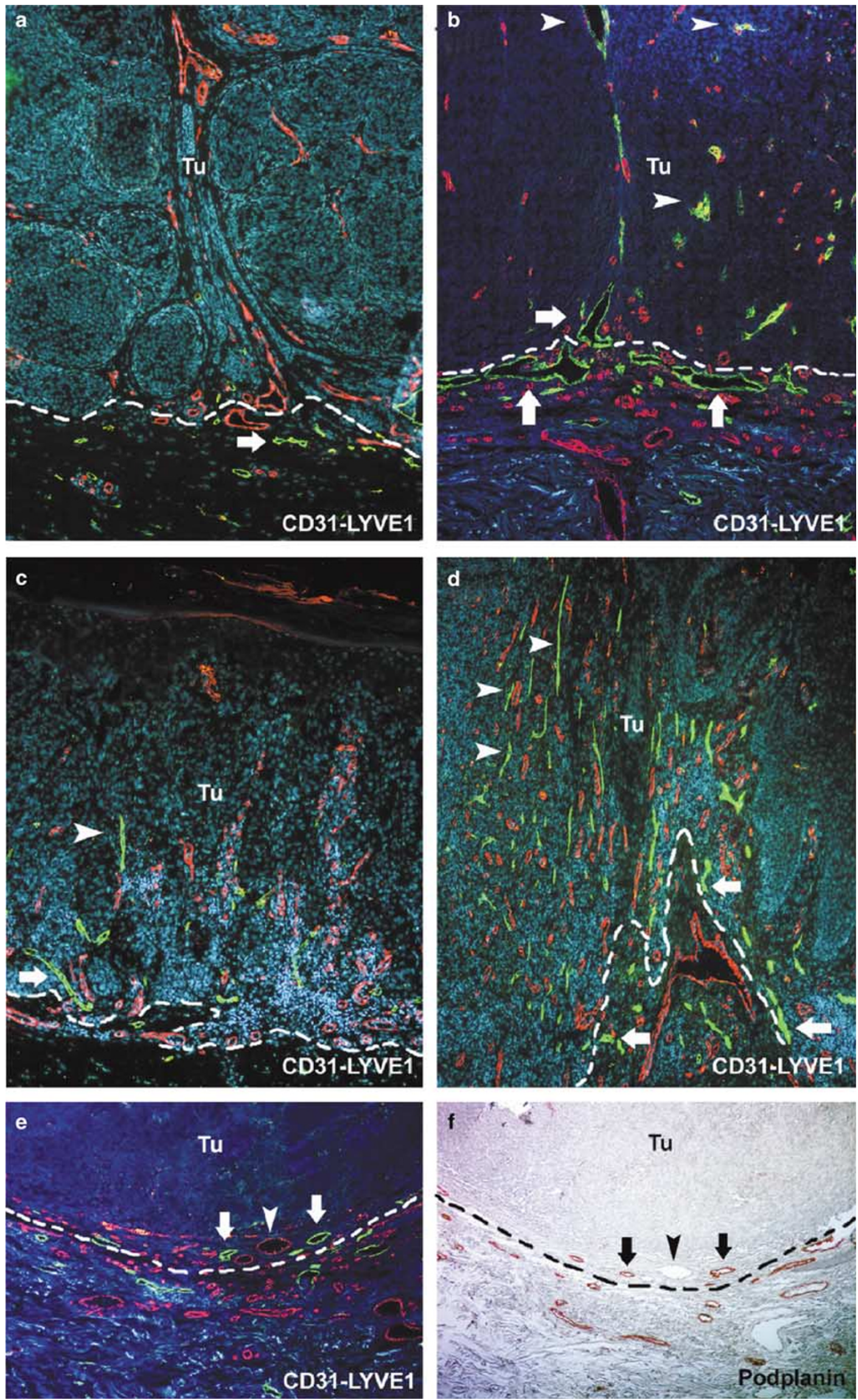
models, we analyzed the expression levels of these proteins in all primary melanomas by immunohistochemistry. We detected moderate to high levels of cytoplasmic VEGF-C in the majority of SLN-positive melanomas but in a minority of the SLN-negative melanomas (Figure 3a-d). In total, 88.2\% of SLNpositive melanomas expressed VEGF-C protein, compared to only $44.0 \%$ of SLN-negative tumors $(P=0.00818$; Table 3$)$. Although the majority of melanoma cells examined contained VEGF-D in
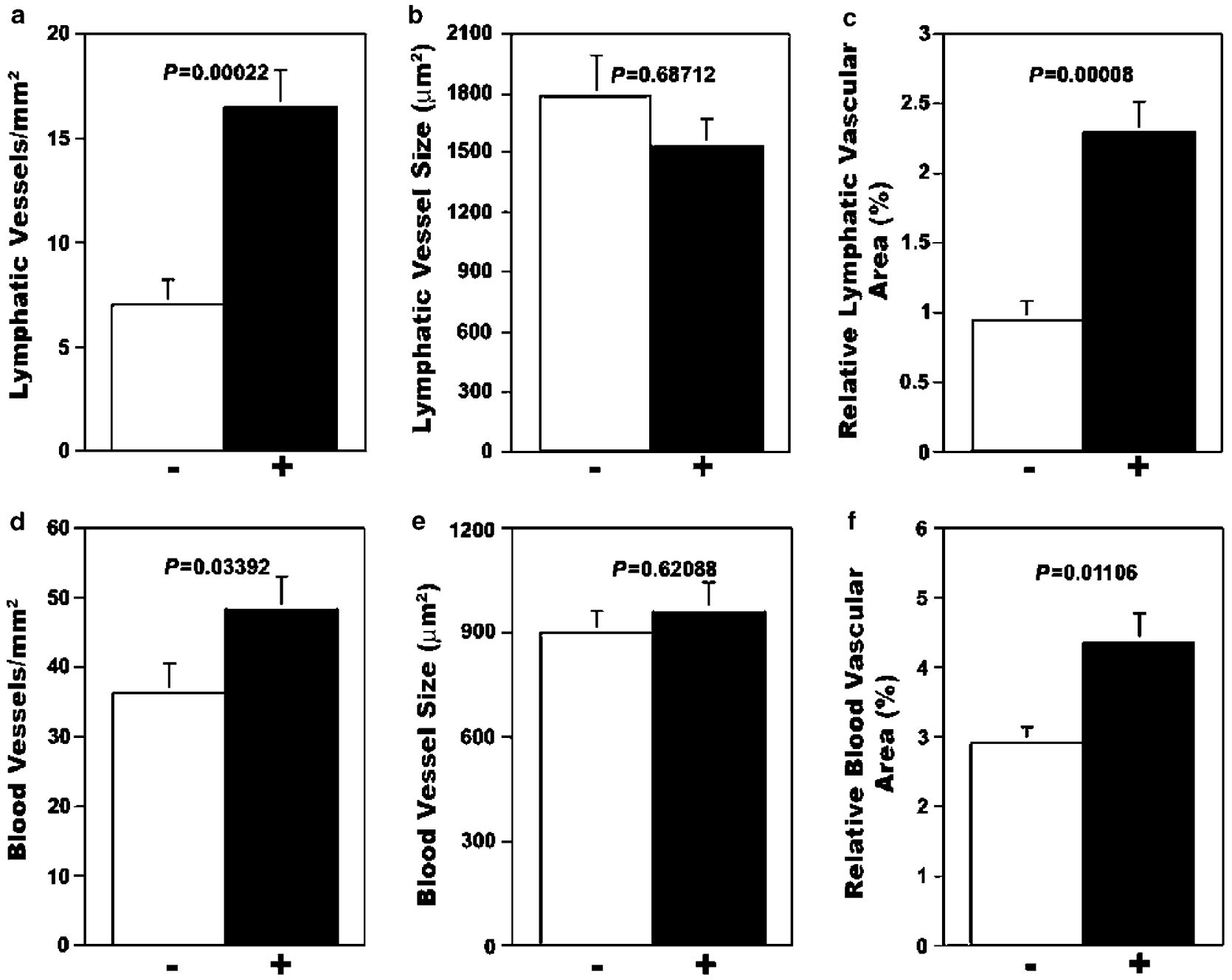

Figure 2 Computer-assisted morphometric analysis of tumor-associated blood and lymphatic vessels in cutaneous melanoma patients with SLN dissection. Panels a-c show the dramatic increase of peritumoral lymphatic vascular density and relative area occupied by lymphatics in SLN-positive melanomas (+; black bars, $n=17)$, compared with SLN-negative melanomas $(-$; white bars, $n=28)$. No change of the size of individual lymphatic vessels was detected. Panels $\mathbf{d}-\mathbf{f}$ show a slight increase of BVD and relative area occupied by blood vessels detected in SLN-positive melanomas, compared to those patients with negative SLN. No change of the size of individual blood vessels was detected. Data are shown as mean \pm s.e.m.

Figure 1 Increased intra- and peritumoral lymphatic density in cutaneous melanomas that have undergone metastasis to SLN. Panels a-e show sections of melanomas that were analyzed by immunofluorescence analysis using antibodies against LYVE-1 (green), which is a lymphatic marker, and CD31 (red) which is panvascular marker. Panels a and c show that in two representative SLN-negative melanomas, only a few lymphatic vessels surrounding (arrows) and inside (arrowhead) the tumor (Tu) border (dotted line). Panels b and d show, in comparison, that SLN-positive melanomas contain prominent 'hot spots', or areas of increased LVD, within (arrowheads) and surrounding (arrows) the tumor border (dotted line). In contrast, blood vessels (red) are homogeneously distributed throughout the tumor. Panel e also shows the increased peritumoral LVD (arrows) at the border of a tumor removed from an SLN-positive patient. A blood vessel, which is LYVE-1 $1^{-}$but CD $31^{+}$, is indicated by the arrowhead. Panel $\mathbf{f}$ shows the results of immunohistochemical analysis of a serial tumor section with the lymphatic-specific antibody D2-40. This antibody has a strikingly similar staining pattern to that of the anti-LYVE-1 antibody (e), labeling tumor-associated lymphatic vessels (arrows) but not blood vessels (arrowhead). Original magnifications: $\times 100$. 
Table 3 Univariate analysis of different risk factors for melanoma metastasis to SLN

\begin{tabular}{|c|c|}
\hline Variable & $\mathrm{P}$-value \\
\hline \multicolumn{2}{|l|}{ Wilcoxon rank test } \\
\hline Relative lymphatic vascular area (\%)a & 0.00008 \\
\hline Lymphatic vessels $/ \mathrm{mm}^{2 \mathrm{a}}$ & 0.00022 \\
\hline Level of invasion $(I V-V)^{b}$ & 0.00010 \\
\hline Tumor thickness (mm) ${ }^{\mathrm{a}}$ & 0.00500 \\
\hline Relative blood vascular area $(\%)^{\mathrm{a}}$ & 0.01106 \\
\hline Blood vessels $/ \mathrm{mm}^{2 \mathrm{a}}$ & 0.03392 \\
\hline Age (years) ${ }^{\mathrm{a}}$ & 0.97243 \\
\hline Lymphatic vessel size $\left(\mu \mathrm{m}^{2}\right)^{\mathrm{a}}$ & 0.68712 \\
\hline Blood vessel size $\left(\mu \mathrm{m}^{2}\right)^{\mathrm{a}}$ & 0.62088 \\
\hline \multicolumn{2}{|l|}{ Fisher's exact test } \\
\hline VEGF-C protein expression ${ }^{\mathrm{b}}$ & 0.00818 \\
\hline VEGF-D protein expression ${ }^{\mathrm{b}}$ & 1.00000 \\
\hline $\operatorname{Sex}^{\mathrm{b}}+1$ & 0.24120 \\
\hline Ulceration $(\mathrm{mm})^{\mathrm{b}}$ & 0.16563 \\
\hline Lymphatic vascular invasion $^{\mathrm{b}}$ & 0.15455 \\
\hline Intratumoral lymphatics ${ }^{\mathrm{b}}$ & 0.11070 \\
\hline Microsatellites $^{\mathrm{b}}$ & 1.00000 \\
\hline \multicolumn{2}{|l|}{$\chi^{2}$} \\
\hline Anatomic site ${ }^{\mathrm{a}}$ & 0.34244 \\
\hline Regression $^{\mathrm{a}}$ & 0.7153 \\
\hline Histologic type $^{\mathrm{a}}$ & 0.20090 \\
\hline Tumor infiltrating lymphocytes ${ }^{\mathrm{a}}$ & 0.88914 \\
\hline
\end{tabular}

${ }^{\mathrm{a}}$ Categorical variables.

${ }^{\mathrm{b}}$ Dichotomous variables.

focal to diffuse cytoplasmic patterns (Figure 3e,f), there was no correlation between VEGF-D levels and metastasis to SLN ( $P=1.00$; Table 3). Overall, these results indicate that VEGF-C, but not VEGF-D, mediates tumor-associated lymphangiogenesis during melanoma metastasis.

\section{Metastasized Melanoma Cells Maintain Lymphangiogenic Activity in SLNs}

To investigate whether the melanoma cells maintain their lymphangiogenic activity once they spread to the SLNs, all tumor-negative $(n=27)$ and -positive SLN $(n=18)$ sections were immunostained for LYVE-1/CD31. Immunofluorescence analysis of normal lymph nodes revealed that the medullary sinuses and hilar efferent lymphatics were LYVE-1positive, whereas the high endothelial venules were LYVE-1-negative (Figure 4a,b). Importantly, 40\% of the metastasized melanomas demonstrated prominent 'hot spots' of lymphatic vessel formation in the SLN (Figure 4d-f). The primary tumors in these patients had a significantly higher LVD (21.86 vessels $/ \mathrm{mm}^{2}$ ) than those that were not associated with lymphangiogenic metastases (13.76 vessels/ $\mathrm{mm}^{2}, P=0.01202$, Figure 4c). These results reveal a positive correlation between tumor lymphangiogenesis in primary melanomas and the lymphangiogenic activity of SLN metastases.

\section{Discussion}

When patients are diagnosed with primary cutaneous melanoma, determining their risk of developing metastases poses a challenge to clinicians and pathologists alike. The early identification of metastatic disease is important, as it determines the requirement for adjuvant therapy and further management. ${ }^{15}$ Prognosis is currently based on tumor thickness, ${ }^{16}$ but a considerable number of patients with thin melanomas also go on to develop metastatic disease. ${ }^{5}$ SLN biopsy is also commonly used to detect metastases in patients with primary melanoma. ${ }^{4}$ It can be a challenge, however, to detect SLN metastases, as they are often microscopic, requiring examination of multiple SLN sections. Dissection of SLN can also result in mild to moderate degrees of lymphedema ${ }^{17}$ or sensory morbidity. ${ }^{18}$ Alternative early-stage prognostic factors are therefore urgently needed.

The discovery of the lymphatic endothelial hyaluronan receptor-1 (LYVE-1) as a specific marker for both normal ${ }^{8}$ and tumor-associated lymphatics, ${ }^{19}$ has facilitated analysis of the association between tumor lymphangiogenesis and clinical outcome. Our study of 45 patients with cutaneous melanoma reveals that quantification of primary tumor lymphangiogenesis can be used to accurately predict which patients are most likely to develop SLN metastases.

Evaluation of tumor lymphangiogenesis can also be used to predict the risk of tumor metastasis beyond the SLN-six out of 18 SLN-positive patients developed distant lymph node and/or organ metastases, and five of these patients (83\%) had an LVA $>1.5 \%$. Furthermore, in patients whose primary melanoma lymphangiogenesis index (LVA) was above $2 \%$, the incidence of melanoma metastasis to SLN was $100 \%$. Another important finding of this study was that an increase in LVD in primary melanomas was correlated with lymphangiogenesis in SLN metastases. Thus, metastatic primary tumor cells maintain their lymphangiogenic activity after they have spread to the lymph nodes. Taken together, these results indicate that lymphangiogenesis induction is an active part of human tumor metastasis, ${ }^{20,21}$ as opposed to the traditional view of a passive role for the lymphatic endothelium during tumor lymphatic invasion and metastasis. ${ }^{22}$

The fact that we detected significantly higher levels of VEGF-C expression in SLN-positive melanoma cells than SLN-negative tumor cells, and that VEGF-C expression was also correlated with the extent of peritumoral lymphangiogenesis, indicates that this growth factor promotes melanoma lymphangiogenesis and metastasis. In fact, tumor cell expression of VEGF-C alone was shown by univariate analysis to predict metastasis to the SLNs. A correlation between VEGF-C mRNA levels and melanoma metastasis to lymph nodes was previously reported. ${ }^{23,24}$ However, the importance of 

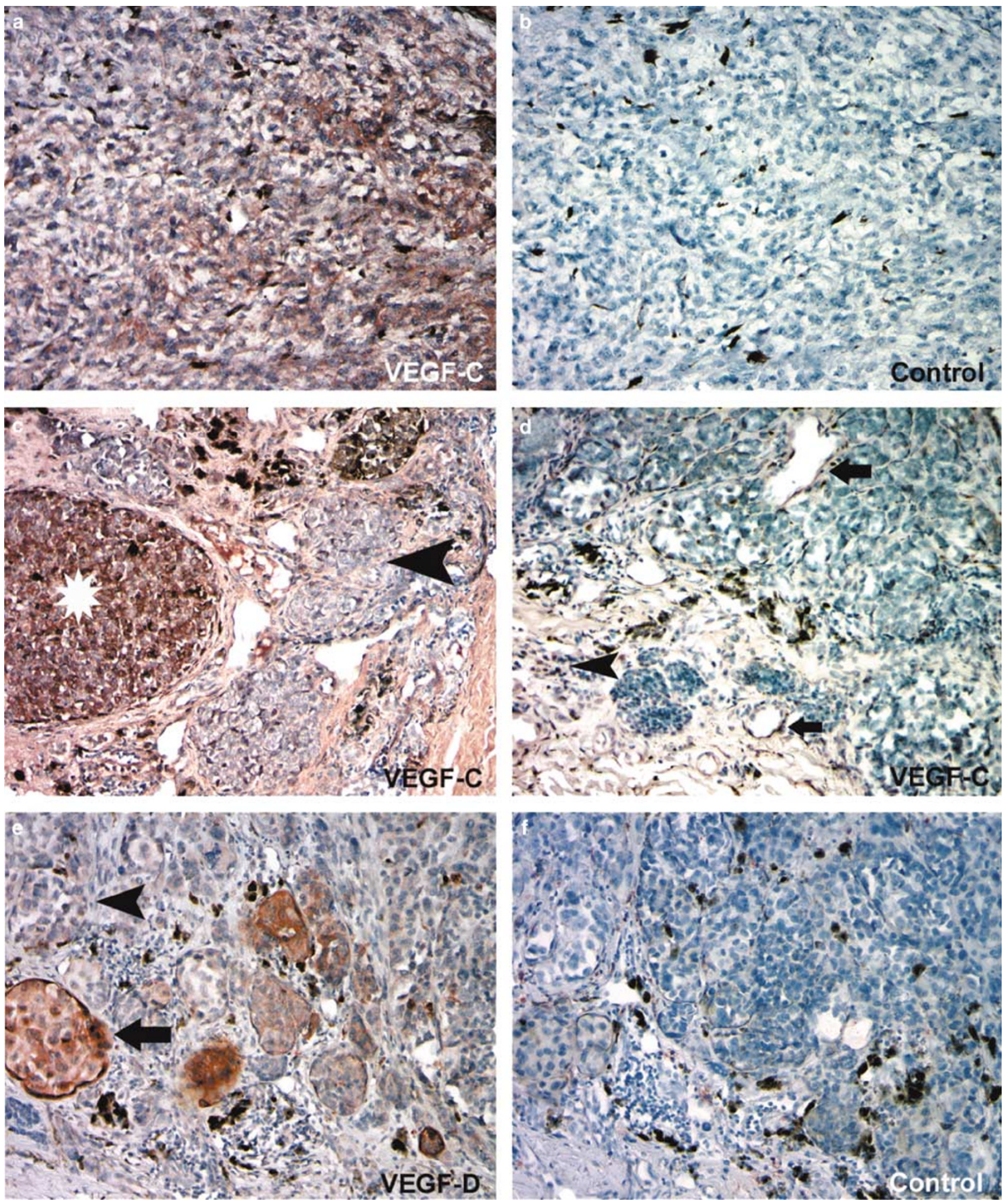

Figure 3 VEGF-C expression in metastatic tumor cells. Panels a-d show representative samples of immunoperoxidase staining of melanomas with an antibody against VEGF-C (red). Panel a shows strong VEGF-C immunoreactivity in the cytoplasm of melanoma cells, whereas panel $\mathbf{b}$ demonstrates lack of staining in the negative control. In panel $\mathbf{c}$, the focal expression of VEGF-C can be detected in the cytoplasm of SLN-positive melanoma cells, in one tumor nest (asterisk) but not in the adjacent nests (arrowhead). Panel d shows that in a representative section of an SLN-negative melanoma, VEGF-C is expressed by macrophages (arrowhead) and blood vascular endothelial cells (arrows), but not by tumor cells. In panel e, immunoperoxidase staining of an SLN-positive sample with an antibody against VEGF$\mathrm{D}$ (red) reveals its focal expression in the cytoplasm of some tumor nests (arrow), but not in others (arrowhead). Panel $\mathbf{f}$ is the matched negative control. Original magnifications: $\times 200$. 

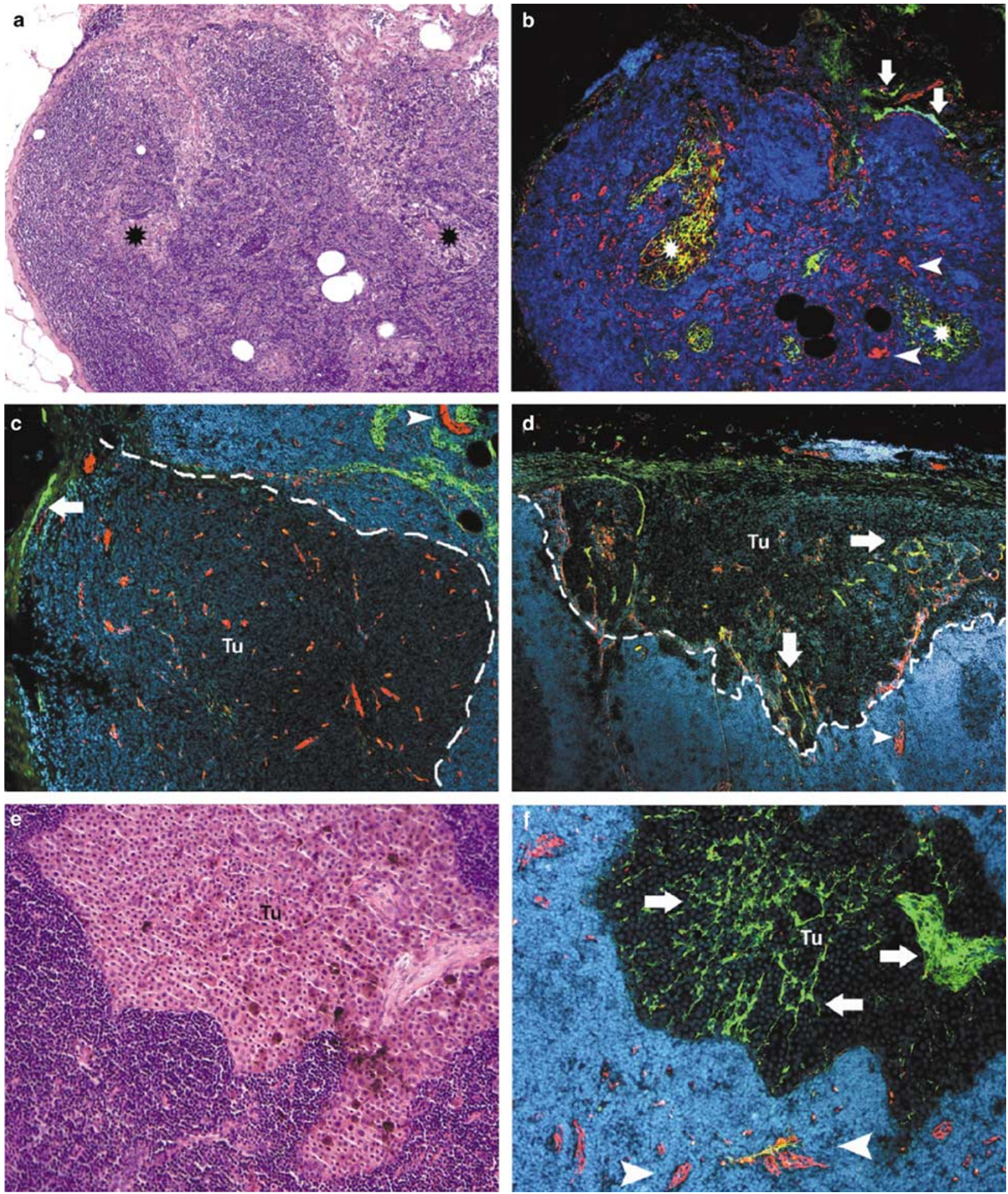

Figure 4 Lymphangiogenesis in melanomas that have metastasized to the SLN. Panel a shows the typical histology of an SLN that does not contain metastatic tumor cells. Medullary sinuses are indicated with asterisks. Panel b shows double immunofluorescence staining of a serial section of the tumor-free lymph node shown in panel a with antibodies against LYVE-1 (green) and against CD31 (red). LYVE-1 medullary sinuses are indicated with asterisks and efferent lymphatics in the hilus are indicated with arrows. In panel c, double immunofluorescence analysis with antibodies against LYVE-1 and CD31 reveals lack of lymphatics within a melanoma that has metastasized to the SLN, except for the afferent lymphatic (arrow) in the capsule. Panel d shows that in contrast, some metastasized melanomas contain prominent 'hot spots' of lymphatic vessels (arrows). Blood vessels are homogeneously distributed throughout the metastasized tumors (Tu) shown in panels $\mathbf{c}$ and $\mathbf{d}$. In panel e, histological analysis of a tumor (Tu) that has metastasized to the SLN reveals its paracortical location. In panel f, double immunofluorescence of a serial section of the tumor shown in panel e, with antibodies against LYVE-1 and CD31, reveals numerous lymphatics (arrows) within the metastasized tumor (Tu). High endothelial venules (arrowheads) are CD $31^{+}$but LYVE-1 ${ }^{-}$. Original magnifications: $\times 100$ 
tumor cell-derived VEGF-C vs VEGF-C produced by tumor-associated macrophages ${ }^{25,26}$ has remained unclear. Our study shows that tumor cells themselves make a significant contribution to VEGF-C production and, therefore, to tumor lymphangiogenesis. We also detected a slight but statistically significant increase in levels of tumor angiogenesis in SLN-positive melanomas. Our finding that the extent of tumor angiogenesis, unlike lymphangiogenesis, was dependent on tumor thickness further supports the concept that angiogenesis, as demonstrated by other investigators, ${ }^{27}$ is required for tumor growth. However, our results indicate that angiogenesis seems to play a lesser role in promoting melanoma metastasis than does the formation of tumor-associated lymphatic vessels.

This is the first study to show that measuring the extent of tumor-associated lymphangiogenesis can serve as a powerful tool for identifying primary melanomas that are likely to undergo metastasisthe presence of metastases can be determined at the earliest stages of tumor progression, when microscopic tumors have developed in the SLN. If these results will be corroborated in additional studies of larger numbers of patients, this new method might provide a safe and accurate future alternative to SLN biopsy analysis. Our results also demonstrate that it is possible to perform immunofluorescence analysis of CD31 and LYVE-1 levels on routine paraffin sections, thereby facilitating larger, prospective, multi-institutional studies. Evaluating the extent of melanoma lymphangiogenesis would provide oncologists and dermatologists with a safer and more effective means of determining which patients with melanoma require adjuvant therapy and further management, possibly including anti-lymphangiogenic therapy.

\section{Acknowledgements}

We thank Dr DG Jackson for the gift of anti-human LYVE-1 antibody, Dr M Achen for the gift of antihuman VEGF-D antibody, M Constant and L Janes for technical assistance, and $\mathrm{V}$ Schacht and $\mathrm{R}$ Kunstfeld for helpful discussions. This work was supported by NIH/NCI Grants CA69184, CA86410 and CA91861 (MD), by the Susan G Komen Breast Cancer Foundation (MD), by American Cancer Society Program Project Grant 99-23901 (MD), by an NIH Pathology Training Grant (SSD), by the Werner and Klara Kreitz Foundation (BL-A) and by the Cutaneous Biology Research Center through the Massachusetts General Hospital/Shiseido Co. Ltd Agreement (MD).

\section{References}

1 Greenlee R, Hill-Harmon M, Murray T, et al. Cancer Statistics, 2001. CA Cancer J Clin 2001;51:15-36.
2 Hall HI, Miller DR, Rogers JD, et al. Update on the incidence and mortality from melanoma in the United States. J Am Acad Dermatol 1999;40: $35-42$.

3 Weinstock MA. Epidemiology, etiology, and control of melanoma. Med Health R I 2001;84:234-236.

4 Gershenwald JE, Thompson W, Mansfield PF, et al. Multi-institutional melanoma lymphatic mapping experience: the prognostic value of sentinel lymph node status in 612 stage I or II melanoma patients. J Clin Oncol 1999;17:976-983.

5 Kalady MF, White RR, Johnson JL, et al. Thin melanomas: predictive lethal characteristics from a 30-year clinical experience. Ann Surg 2003;238: 528-535; discussion 535-527.

6 Skobe M, Hawighorst T, Jackson DG, et al. Induction of tumor lymphangiogenesis by VEGF-C promotes breast cancer metastasis. Nat Med 2001;7:192-198.

7 Stacker SA, Caesar C, Baldwin ME, et al. VEGF-D promotes the metastatic spread of tumor cells via the lymphatics. Nat Med 2001;7:186-191.

8 Banerji S, Ni J, Wang SX, et al. LYVE-1, a new homologue of the CD44 glycoprotein, is a lymphspecific receptor for hyaluronan. J Cell Biol 1999; 144:789-801.

9 Dadras SS, Paul T, Bertoncini J, et al. Tumor lymphangiogenesis: a novel prognostic indicator for cutaneous melanoma metastasis and survival. Am J Pathol 2003; 162:1951-1960.

10 Clemente CG, Mihm Jr MC, Bufalino R, et al. Prognostic value of tumor infiltrating lymphocytes in the vertical growth phase of primary cutaneous melanoma. Cancer 1996;77:1303-1310.

11 Detmar M, Velasco P, Richard L, et al. Expression of vascular endothelial growth factor induces an invasive phenotype in human squamous cell carcinomas. Am J Pathol 2000;156:159-167.

12 Kahn HJ, Marks A. A new monoclonal antibody, D2-40, for detection of lymphatic invasion in primary tumors. Lab Invest 2002;82:1255-1257.

13 Schacht V, Dadras SS, Johnson L, et al. Upregulation of the lymphatic marker podoplanin, a mucin-type transmembrane glycoprotein, in human squamous cell carcinomas and germ cell tumors. Am J Pathol 2005; 166:913-921.

14 Achen MG, Williams RA, Minekus MP, et al. Localization of vascular endothelial growth factor-D in malignant melanoma suggests a role in tumor angiogenesis. J Pathol 2001;193:147-154.

15 Roberts AA, Cochran AJ. Pathologic analysis of sentinel lymph nodes in melanoma patients: current and future trends. J Surg Oncol 2004;85:152-161.

16 Balch CM, Buzaid AC, Soong SJ, et al. Final version of the American Joint Committee on Cancer staging system for cutaneous melanoma. J Clin Oncol 2001;19: 3635-3648.

17 Wrone DA, Tanabe KK, Cosimi AB, et al. Lymphedema after sentinel lymph node biopsy for cutaneous melanoma: a report of 5 cases. Arch Dermatol 2000; 136:511-514.

18 Temple LK, Baron R, Cody III HS. Morbidity after sentinel lymph node biopsy and axillary dissection: a prospective study of 233 women. Ann Surg Oncol 2002;9:654-662.

19 Beasley NJ, Prevo R, Banerji S, et al. Intratumoral lymphangiogenesis and lymph node metastasis in head and neck cancer. Cancer Res 2002;62:1315-1320. 
20 Detmar M, Hirakawa S. The formation of lymphatic vessels and its importance in the setting of malignancy. J Exp Med 2002;6:713-718.

21 Dadras SS, Detmar M. Angiogenesis and lymphangiogenesis of skin cancers. Hematol Oncol Clin North Am 2004;18(5):1059-1070.

22 Carmeliet P, Jain RK. Angiogenesis in cancer and other diseases. Nature 2000;407:249-257.

23 Goydos JS, Gorski DH. Vascular endothelial growth factor C mRNA expression correlates with stage of progression in patients with melanoma. Clin Cancer Res 2003;9:5962-5967.

24 Schietroma C, Cianfarani F, Lacal PM, et al. Vascular endothelial growth factor-C expression correlates with lymph node localization of human melanoma metastases. Cancer 2003;98:789-797.

25 Skobe M, Hamberg LM, Hawighorst T, et al. Concurrent induction of lymphangiogenesis, angiogenesis, and macrophage recruitment by vascular endothelial growth factor-C in melanoma. Am J Pathol 2001;159: 893-903.

26 Schoppmann SF, Birner P, Stockl J, et al. Tumorassociated macrophages express lymphatic endothelial growth factors and are related to peritumoral lymphangiogenesis. Am J Pathol 2002;161: 947-956.

27 Folkman J. Angiogenesis and tumor growth. N Engl J Med 1996;334:9219. 